\title{
The Designing of a FIR Low Pass Filter and Amplifier for Small Voltage Signals using Kaiser Window
}

\author{
Hemant Kumar Gupta \\ JECRC UDML College of Engineering, \\ Jaipur, Rajasthan, India - 302022
}

\author{
Ritu Vijay,Ph.D
}

Banasthali University, Banasthali,Jaipur, Rajasthan,

\author{
Neetu Gupta \\ Rajasthan College of Engineering \\ for Women, Jaipur, Rajasthan
}

\begin{abstract}
Digital signal processing (DSP) is the study of signals in a digital representation and the processing methods of these signals. A digital filter uses a digital processor to perform numerical calculations on sampled values of the signal. The analog input signal must first be sampled and digitized. The resulting binary numbers, representing successive sampled values of the input signal, are transferred to the processor, which carries out numerical calculations on them. These calculations typically involve multiplying the input values by constants and adding the products together. If necessary, the results of these calculations, which now represent sampled values of the filtered signal, are output to convert the signal back to analog form. To achieve the desired filtering effect, Digital filters can achieve virtually any filtering effect that can be expressed as a mathematical function or algorithm.
\end{abstract}

\section{INTRODUCTION}

In the design of frequency selective filters, desired filter characteristics are specified in the frequency domain in terms of the desired magnitude \& phase response of the filter. In the filter design process, the coefficients of a causal FIR are determined that closely approximates the desired frequency response specifications.

Although the frequency response characteristics possess by ideal filter may be desirable, they are not absolutely necessary in most practical applications if these conditions are relaxed, it is possible to realize causal filters that approximate ideal filters as closely as desired.

In this paper the EMG signals are considered which have the voltage levels in the range of $10 \mathrm{mv}$ and frequency up to $120 \mathrm{~Hz}$ which is considered as the low frequency small voltage signal. Now a low pass FIR filter for the small signal having the cutoff frequency of $120 \mathrm{~Hz}$ is designed.

\section{SYSTEM MODEL}

A desirable property of the window function is that the function is of finite duration in the time domain and that the Fourier transform has maximum energy in the main lobe or a given peak side lobe amplitude. In a Kaiser window the side lobe level can be controlled with respect to the main lobepeak by varying a parameter, $\alpha$. The Kaiser Window function is given by

$w_{k^{(n)}}=\left\{\begin{aligned} \frac{I_{0}(\beta)}{I_{0}(\alpha),} & \text { for }|n| \leq \frac{M-1}{2} \\ 0, & \text { else where }\end{aligned}\right.$

Where

$$
\beta=\alpha\left[1-\left(\frac{2 n}{M-1}\right)^{2}\right]^{0.5}
$$

\section{$I_{0}(\beta) \& I_{0}(\alpha)$ are the Bessel functions}

The actual passband ripple $A_{p}$ and minimum stop band attenuation $\mathrm{A}_{\mathrm{s}}$ are given by

$A_{p}=20 \log _{10} \frac{1+d p}{1-\partial p} \mathrm{~dB}$

As $=-20 \log _{10} \delta_{\mathrm{s}} \mathrm{dB}$

$\delta=\min \left(\delta_{\mathrm{p}}, \delta_{\mathrm{s}}\right)$

From the Kaiser design equation

$\alpha=\left\{\begin{array}{cc}0 & \text { for } A_{x} \leq 21 \\ 0.5842\left(A_{x}-21\right)^{0.4}+0.07886\left(A_{x}-21\right) & \text { for } 21<A_{x} \leq 50 \\ 0.1102\left(A_{x}-8.7\right) & \text { for } A_{x}>50\end{array}\right.$

Order of the filter can be find from the equation $M \geq \frac{F D}{\Delta F}+1$

Where the parameter $\mathrm{D}$ is

$f(x)= \begin{cases}0.9222, & \text { for } A_{g} \leq 21 \\ \frac{A_{g}-7.95}{14.36}, & \text { for } A_{g}>21\end{cases}$

The impulse response is computed from

$\mathrm{h}(\mathrm{n})=\mathrm{w}_{\mathrm{k}}(\mathrm{n}) \mathrm{h}_{\mathrm{d}}(\mathrm{n})$, for $|n| \leq \frac{M-1}{2}$

for Low Pass FIR Filter

$f(x)= \begin{cases}\left(2 \frac{f_{c}}{F}\right) \frac{\sin 2 \pi n f_{d} / F}{2 \pi n f_{c} / F}, & \text { for } n>0 \\ 2 \frac{f_{c}}{F} & \text { for } n=0\end{cases}$

Where $f_{c}=0.5\left(f_{p}+f_{g}\right)$, and $\Delta F=\left(f_{s}-f_{p}\right)$

Algorithms 1

1. Start from initial point $\delta=\min \left(\delta_{\mathrm{p}}, \delta_{\mathrm{s}}\right)$

2. Calculate the minimum stopband attenuation $\left(\mathrm{A}_{\mathrm{s}}\right)$.

3. Compute the parameter $\alpha$ and parameter D

4. Now we calculate the order of filter $M$ which generate the coefficients of low pass FIR filter.

5. These coefficients can also be find out in MATLAB by using filter designing and analysis. 


\section{Coefficient:}

$\mathrm{h}(0)=2.823182709039 \mathrm{e}-005, \mathrm{~h}(1)=-0.0001281807058205$, $\mathrm{h}(2)=-0.0005583885039392, \mathrm{~h}(3)=7.814605953656 \mathrm{e}-019$, $\mathrm{h}(4)=0.002324543544181, \mathrm{~h}(5)=0.002522685109819$, $\mathrm{h}(6)=-0.004154983325905, \mathrm{~h}(7)=-0.01054471968294$, $\mathrm{h}(8)=6.154784193546 \mathrm{e}-018, \mathrm{~h}(9)=0.0234258008186$, $\mathrm{h}(10)=0.02093083214918, \mathrm{~h}(11)=-0.03013912511589$, $\mathrm{h}(12)=-0.07130955821932 \mathrm{~h}(13)=1.413841573615 \mathrm{e}-017$, $\mathrm{h}(14)=0.1948586436646, \mathrm{~h}(15)=0.3727442184403$, $h(16)=0.3727442184403, h(17)=0.1948586436646$, $\mathrm{h}(18)=1.413841573615 \mathrm{e}-017, \mathrm{~h}(19)=-0.07130955821932$, $\mathrm{h}(20)=-0.03013912511589, \mathrm{~h}(21)=0.02093083214918$, $\mathrm{h}(22)=0.0234258008186, \mathrm{~h}(23)=6.154784193546 \mathrm{e}-018$, $\mathrm{h}(24)=-0.01054471968294, \mathrm{~h}(25)=-0.004154983325905$, $\mathrm{h}(26)=0.002522685109819, \mathrm{~h}(27)=0.002324543544181$, $\mathrm{h}(28)=7.81460595365 \mathrm{e}-019, \mathrm{~h}(29)=0.0005583885039392$, $\mathrm{h}(30)=0.0001281807058205, \mathrm{~h}(31)=2.823182709039 \mathrm{e}-005$

\section{Simulated frequency response}

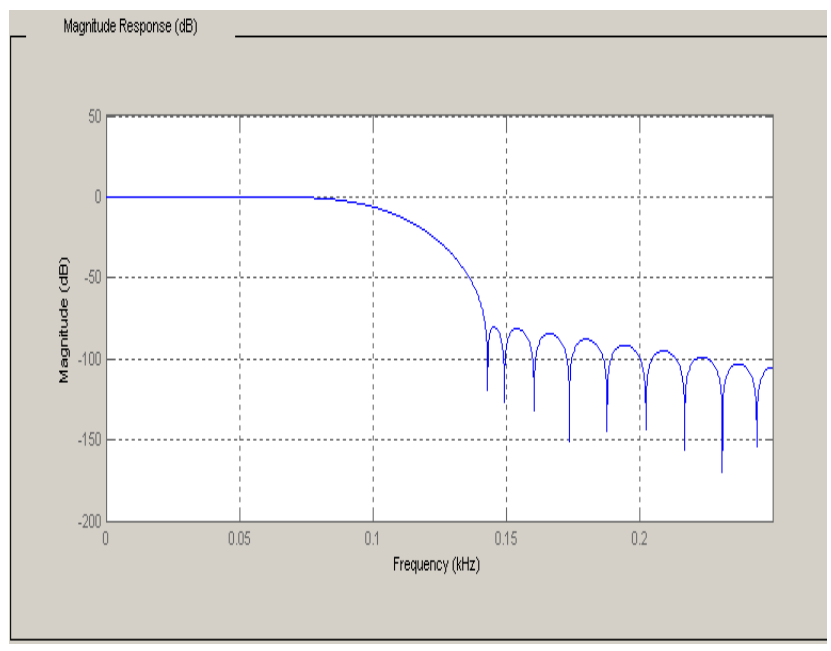

\section{Algorithm2: Real Time Implementation}

1. Initialize the header files such filter files, kit operating files \& input-output line files to include it in the program.

2. Store the analog signal into the buffer registers.

3. Store the filter coefficients into a memory location.

4. Generate the codec for programming at buffering level.

5. Shuffle the buffer according to order of filter.

6. Multiply the filter coefficients with discrete signal values and add it up to the order of the filter.

7. Store the resultant discrete samples and fetch it to the output section.

Note: since used order of the filter as 31 and the input signal is having the voltage level $6.8 \mathrm{mV}$ hence the output after filtering is being attenuated with a factor $1 / 3$ and the signal will disappear in real time application so the need of amplification occurs.

\section{Algorithm3: Real Time Implementation}

1. The applied analog signal is digitized through DSP processor

2. These digitized samples are passed through the multiplication factor of gain of amplifier

3. The gained signal is factorized with the coefficient of the order of the filter.

4. The amplification factor depends on the requirements and be so chosen that it should not disturbed the shape of output means the noise level should be minimum.

5. These amplified samples stored into the memory location of the processor.
International Journal of Computer Applications (0975 - 8887)

Volume 74-No.6, July 2013

6. Stored samples are multiplied and add with the coefficients of the filter number of times according to the order of filter.
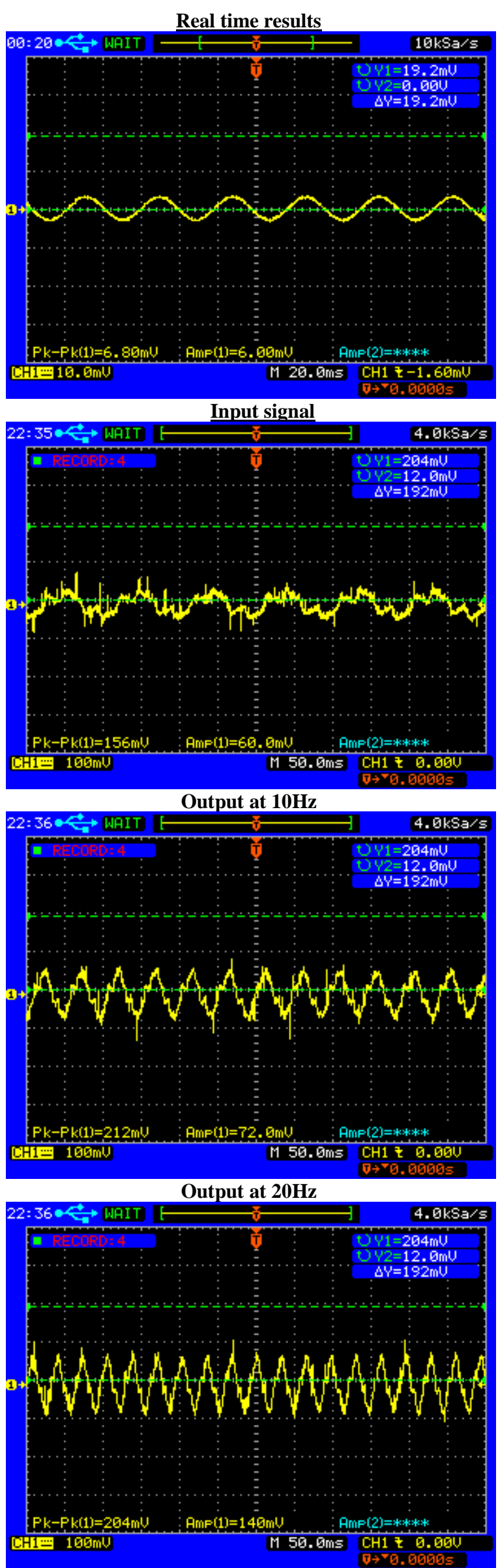
Output at 30Hz
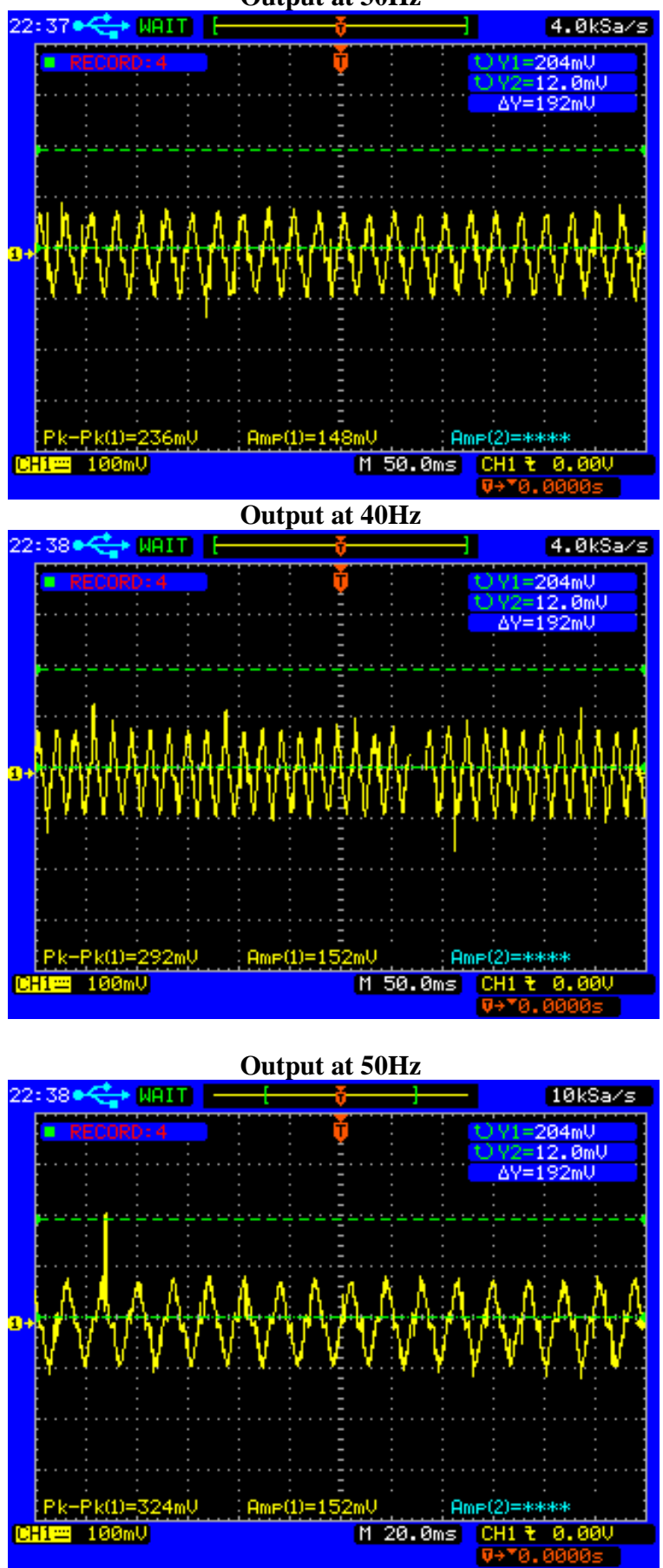

\section{Output at $60 \mathrm{~Hz}$}

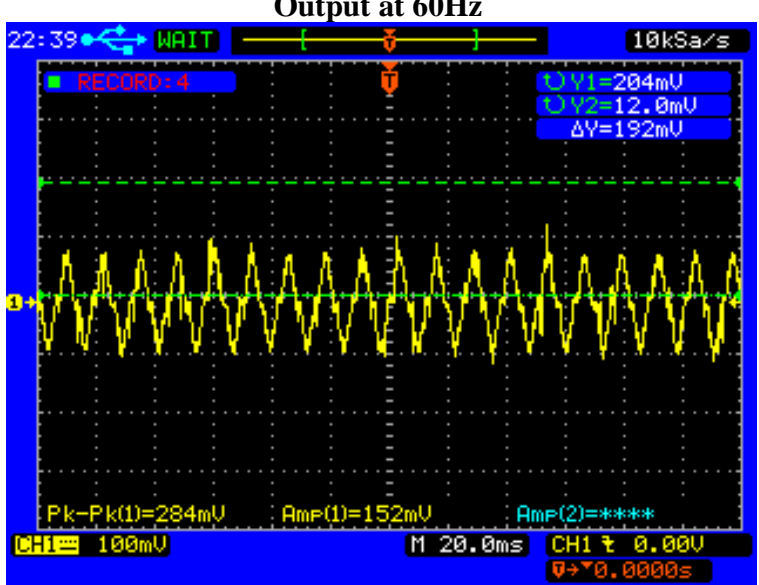

Output at $70 \mathrm{~Hz}$

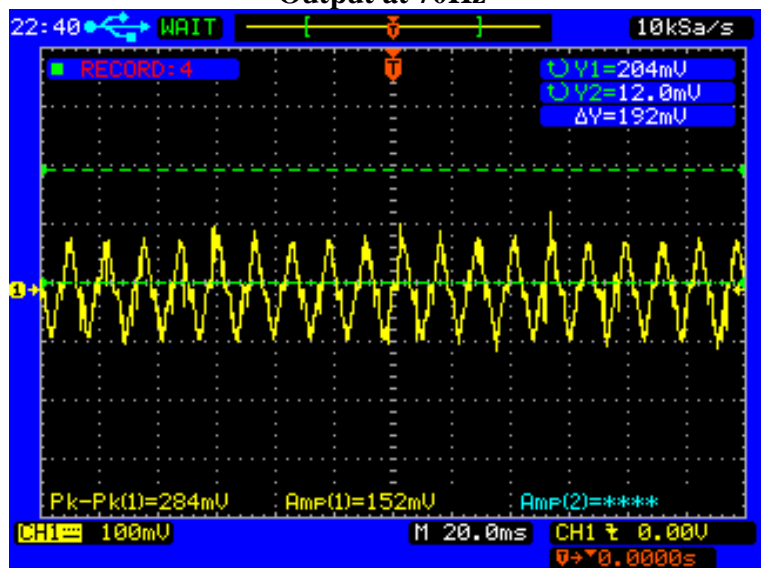

\section{Output at $80 \mathrm{~Hz}$}

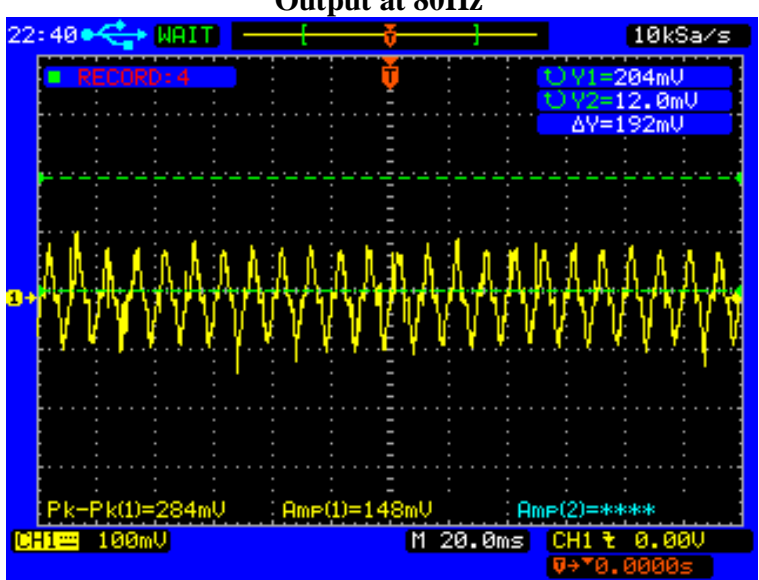

Output at $90 \mathrm{~Hz}$

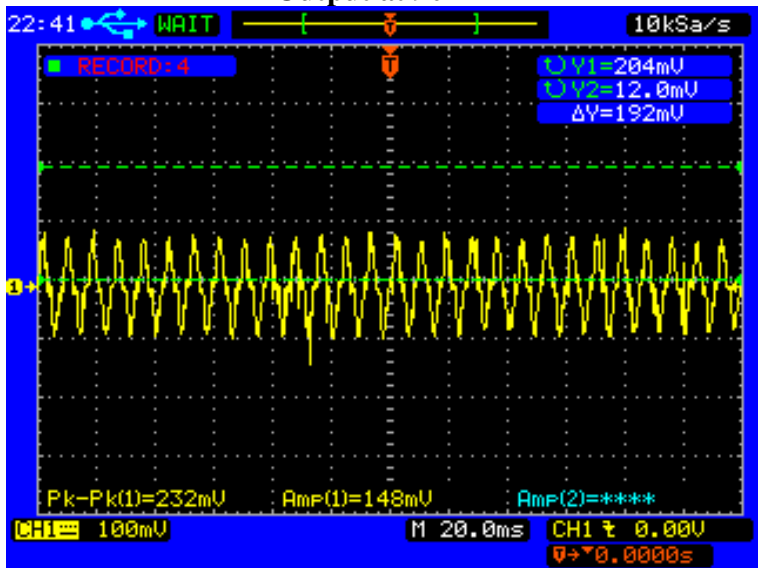

\section{Output at $100 \mathrm{~Hz}$}

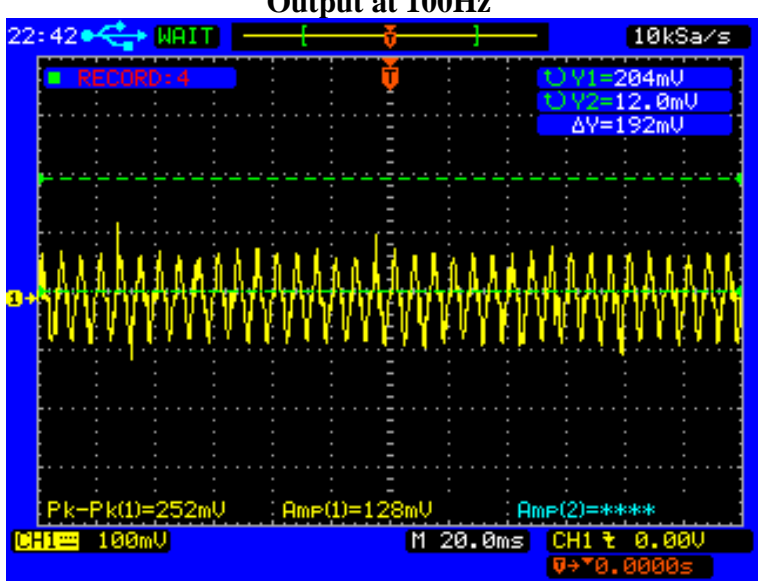



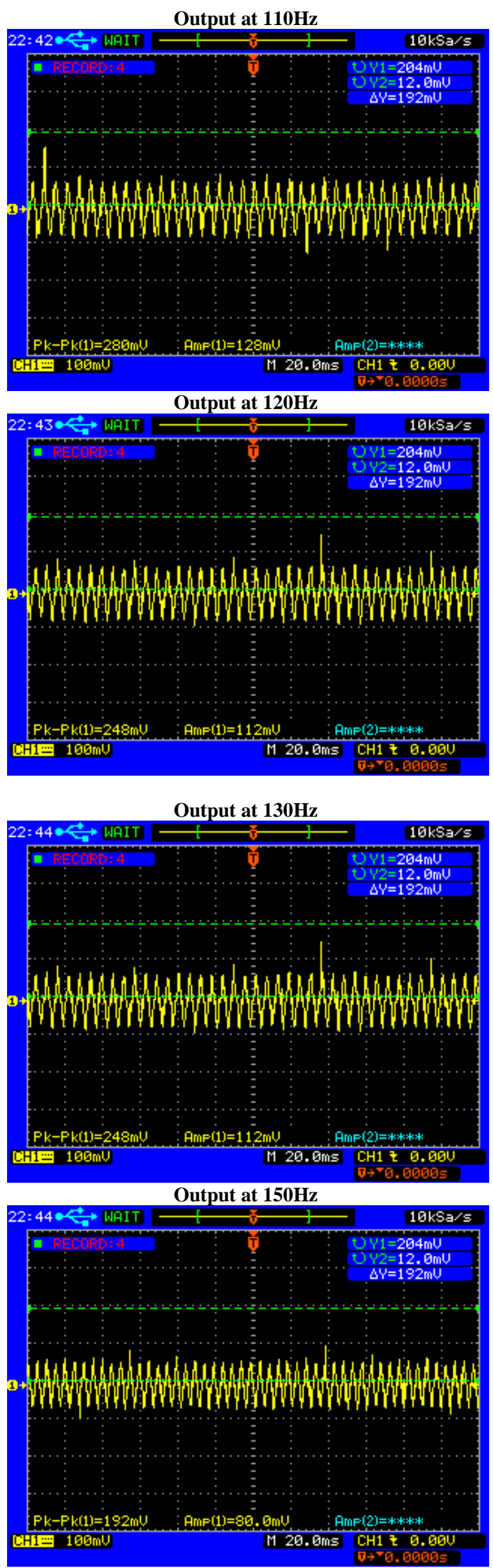

Output at $200 \mathrm{~Hz}$

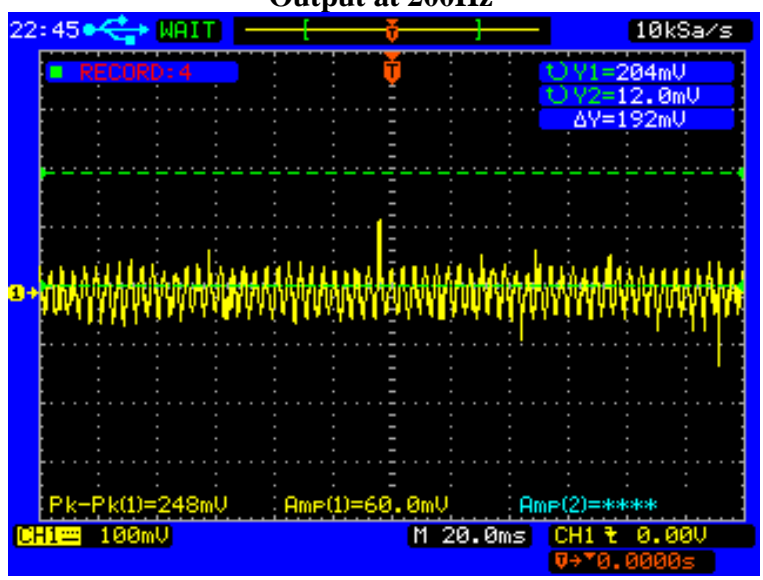

Output at $400 \mathrm{~Hz}$

Input Simulated EMG Signal

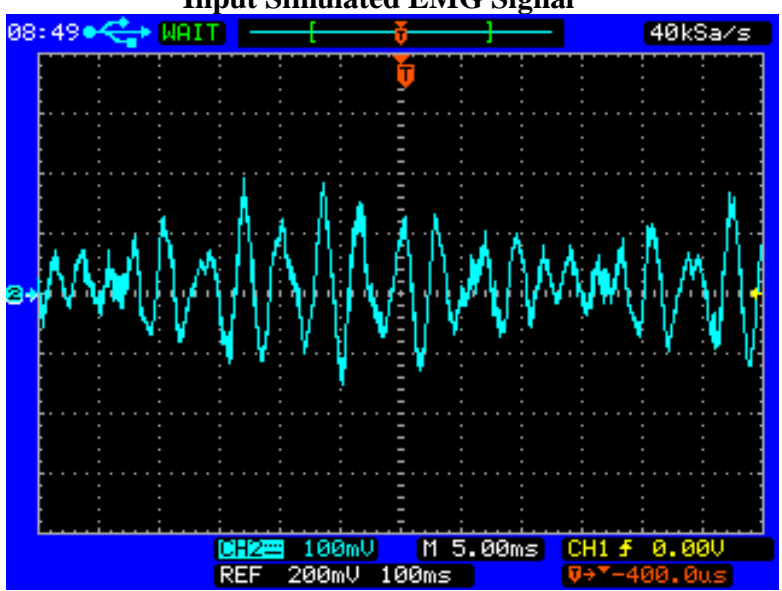

Output correspond to Simulated EMG Signal

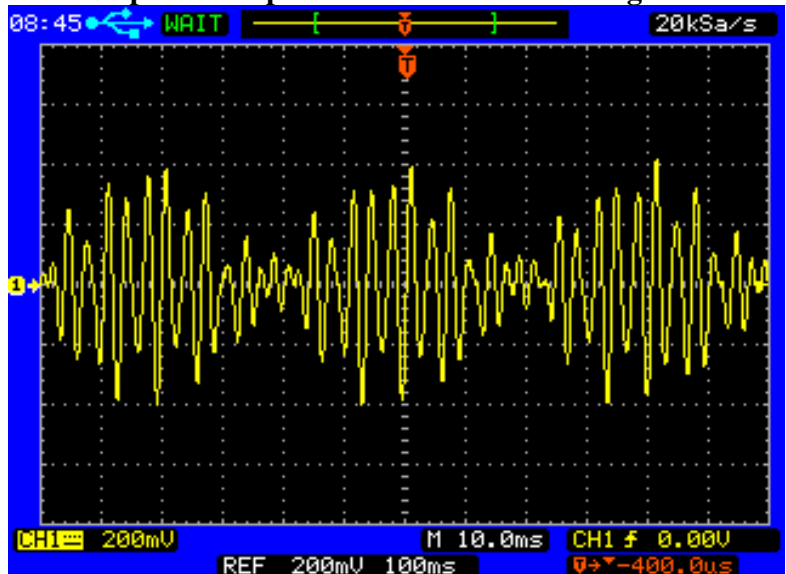

Input Actual Bicep EMG Signal

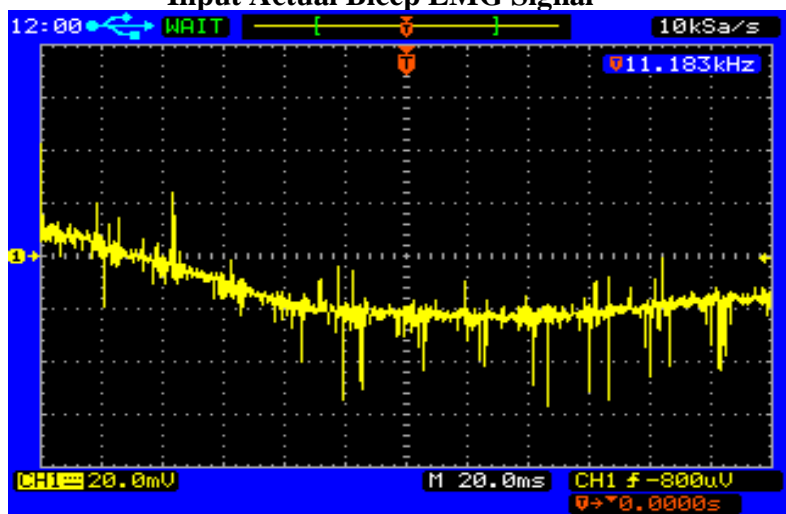


Output correspond to Actual Bicep EMG Signal

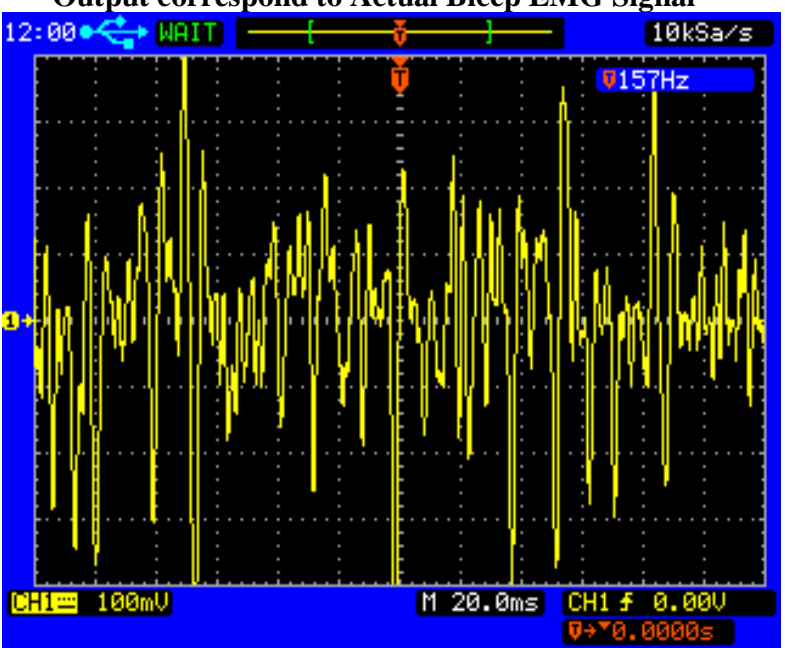

Input Actual extensor carpi radialislongus EMG Signal

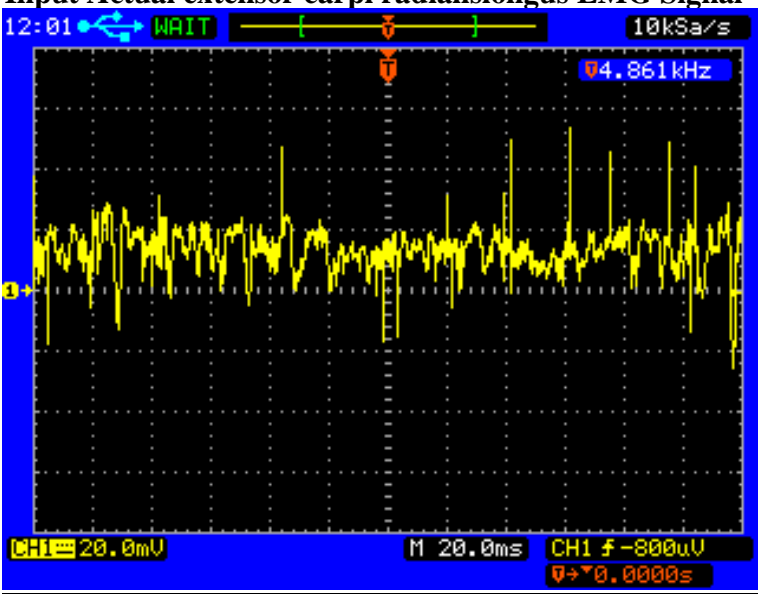

Output correspond to Actual extensor carpi radialislongus EMG Signal

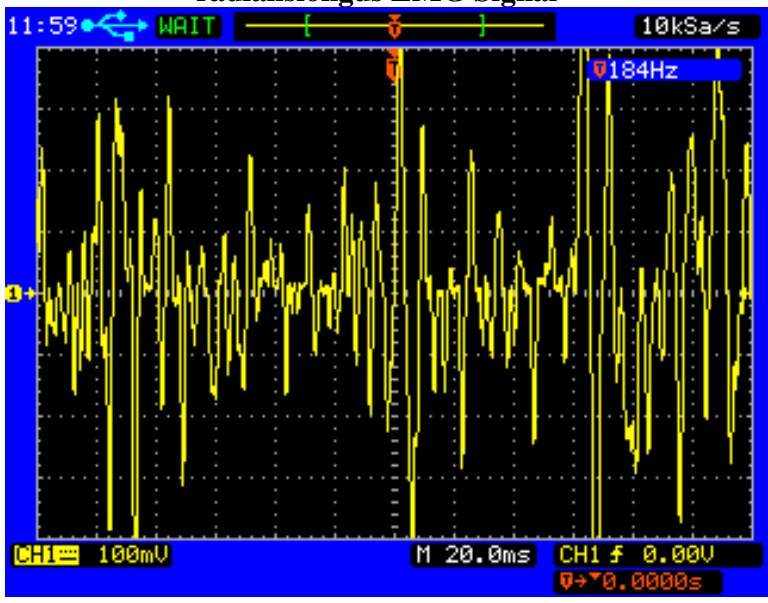

\section{ANALYSIS}

Ffrom the comparison of simulated result \& real time results, the frequency response is nearly identical \& efficient. The proposed digital FIR low pass filter and amplifier can be extensively used in bio-medical engineering field. This can also be used in précised equipment manufacturing for low signal analysis. The proposed method is highly effective in analyzing the EMG signals. It is easy to use \& apply in real time processing of any random signal obtained from the bio-medical sensors.

\section{FUTURE SCOPE:}

Digital signal processing is a rapid growing field; the most of work in signal processing is being digitized for the accessibility and the reliability of digital signal processing. It is good era to work in this field for the research scholars. The key factor in digital signal processing is the filter designing to meet the requirements in various applications.

\section{CONCLUSION}

In this paper, a digital FIR low pass filter having cutoff frequency $120 \mathrm{~Hz}$ applicable on very small voltage signal like EMG is designed. The faithful amplification is also been achieved through the digital amplification process. From result studies, the proposed method is highly effective \& efficient.

\section{REFERENCES:}

[1] A New Structure Of Sharp Transition FIR Filters Using Frequency-Response Masking, Ronghuan Yang, Bede Liu, Fellow, Ieee, And Yong Ching Lim, Member, Ieee

[2] Time Series Calculation Of Heart Rate Using Multi Rate FIR Filters MR Risk1, DF Slezak1, P Turjanski1, A Panelli1, RAM Taborda2, G Marshall1 1CONICET And Computer Science Department, Fceyn University Of Buenos Aires, Argentina, 2LIADE, Fcefyn National University Of C'Ordoba, Argentina

[3] Design Of Optimal Finite Word Length FIR Digital Filters Using Integer Programming Techniques, U`SAN M. KODEK, MEMBER, IEEE

[4] Comparison Of Filter Design Methods To Generate Analytic Signals Laurent Vanbeylen, Johan Schoukens, Vrije Universiteit Brussel, Dep. ELEC, Pleinlaan, 21050 Brussels, BELGIUM E-Mail: Laurent.Vanbeylen@Vub.Ac.Be

[5] Design Of Fractional Delay Filters Using Convex Optimization, William Putnam Julius Smith , Department Of Electrical Engineering And Center For Research In Music And Acoustics (CCRMA) Stanford University Stanford, CA 94305-8180

[6] Low Complexity FIR Filters Using Factorization Of Perturbed Coefficients $\dagger$, Cassondra Neau, Khurram Muhammad*, And Kaushik Roy ECE, Purdue University, West Lafayette, IN 47907 *Texas Instruments, Dallas, TX 
[7] Hamilton-Wright \& D.W. Stashuk, Physiologically Based Simulation Of Clinical EMG Signals, Vol. 52, Pp 171-183, February 2005.

[8] F. Odille, C. Pasquier, R. Asbacherli, P.A. Vuissoz, G.P Zientara \& J. Feblinger, Noise Cancellation Signal Processing Method \& Computer System For Improved Real Time ECG Artifact Correction During MRI Data Acquit Ion, Vol. 54, Pp 630-639, April 2007.

[9] Farid Mobasser, J.Mikael Eklund, Member IEEE \& Keyvan Hashtrudi-Zaad, Member IEEE, "Estimation
Of Elbow Induced Wrist Force With EMG Signals Using Fast Orthogonal Search", Vol. 54, Pp 543-579, April 2007.

[10] Z.G. Feng \& K.L. Teo, “A Discrete Filled Function Method For The Design Of FIR Filters With Signed Powers Of Two Coefficients", Vol. 56, Pp 134-139, January 2008.

[11] Low Power Asynchronous Digital Signal Processing, A Thesis Submitted To the University Of Manchester For The Degree Of Doctor Of Philosophy In The Faculty Of Science \& Engineering 